\title{
Seeing the Atoms More Clearly: STEM Imaging from the Crewe Era to Today
}

\section{S.J. Pennycook}

Materials Science and Technology Division, Oak Ridge National Laboratory, Oak Ridge, TN

When Albert Crewe and his coworkers successfully developed the high brightness cold field emission gun [1] it brought the scanning transmission electron microscope (STEM) into the modern era, capable, for the first time, of high resolution imaging and electron energy loss spectroscopy [1]. Crewe realized that the STEM geometry separated the probe-forming optics from the detector optics, bringing great flexibility, efficient signal collection and the possibility of multiple simultaneous signals. A few years later, the first electron microscope images of single atoms were obtained using an annular detector [2, 3]. Heavy atoms were supported on thin carbon films and the resolution achieved was about $2.5 \AA$. The first Z-contrast images were obtained by using the ratio of the annular dark field (ADF) signal (dominated by elastic scattering and proportional to $Z^{3 / 2}$ ) to the inelastic signal obtained from the spectrometer (mostly passing through the hole in the annular detector and proportional to $\mathrm{Z}^{1 / 3}$ ). Crewe also mentions how the STEM is ideally suited to aberration correction since it can be applied before the sample introduces an energy spread into the beam.

These spectacular innovations generated great excitement and commercial STEMs soon became available from VG Microscopes [4] with a resolution of about $5 \AA$. Attempts to use the Crewe ratio method in crystalline materials were disappointing, however, as any Z-contrast contrast could be obscured by diffraction contrast. This led to attempts to go to higher detection angles, to detect thermal diffuse scattering and minimize diffraction contrast [5-7], the first high angle annular dark field (HAADF) images. When higher resolution pole pieces became available for the STEM, atomic resolution images could be obtained either in an ADF image [8] or a HAADF image [9-11]. The images showed all the characteristics of incoherent images, just as the single atom images had done, which was explained through a Bloch wave analysis that showed how most of the high angle scattering came from 1s type Bloch states localized on atomic columns. Contributions from other Bloch states were minimal, so that dynamical thickness oscillations were not seen $[12,13]$. The contrast was predominantly $Z$-contrast ( $\mathrm{Z}^{2}$ at sufficiently high angles), did not reverse with thickness or defocus, and the image showed higher resolution than a phase contrast image, characteristics that allowed direct interpretation at atomic resolution. It was not long before the possibility of simultaneous HAADF and EELS signals was used to demonstrate atomically-resolved spectra, locating the probe on planes [14] or single atomic columns [15] identified in the image.

Nevertheless, STEM images remained noisy relative to TEM images until the era of aberration correction, which not only improved image resolution, but also signal to noise ratio. Clear images of single atoms were achieved [16], sub-Ångstrom lattice spacings were resolved [17], and single atoms inside crystals were identified by EELS [18]. The introduction of $5^{\text {th }}$ order correctors brought further gains in image resolution $[19,20]$ and high efficiency two-dimensional elemental mapping in EELS [21, 22] and even with X-rays [23, 24]. Light atoms were imaged and identified using a wide angle annular detector to maximize collection efficiency, exactly the strategy used originally by Crewe. The reduced probe size, $\sim 1.2 \AA$ at $60 \mathrm{kV}$, allowed individual B, C, N and $\mathrm{O}$ in monolayer $\mathrm{BN}$ to be resolved and identified based on image intensity [25]. We have also seen the introduction of annular bright field imaging proposed originally by Rose [26]. It combines the high sensitivity of 
a phase contrast image with the (largely) incoherent characteristics of a Z-contrast image [27-29], enabling the imaging of hydrogen columns in $\mathrm{VH}_{2}$ and $\mathrm{YH}_{2}[28,30]$. Aberration correction should also allow the correlation of atomic resolution images and spectroscopy with signals that map functionality, such as cathodoluminescence for mapping optical emission in light emitting materials and electron beam induced current for mapping carrier generation efficiency in solar cell materials.

It is clear that the successful correction of aberrations has transformed the STEM. By enabling highly efficient and flexible atomic resolution imaging and spectroscopy, Crewe's original vision has been brought to a practical reality.

References

[1] A. V. Crewe, Science 154, 729 (1966).

[2] A. V. Crewe, J. Wall, and J. Langmore, Science 168, 1338 (1970).

[3] J. Wall et al., Proc. Natl. Acad. Sci. U. S. A. 71, 1 (1974).

[4] I. R. M. Wardell, and P. E. Bovey, in Advances In Imaging And Electron Physics, edited by P. W. Hawkes (Elsevier, 2009), pp. 221.

[5] A. Howie, J Microsc-Oxford 117, 11 (1979).

[6] M. M. J. Treacy, A. Howie, and C. J. Wilson, Philos. Mag. A 38, 569 (1978).

[7] M. M. J. Treacy, A. Howie, and S. J. Pennycook, in Electron Microscopy and Analysis 1979, edited by T. Mulvey (Institute of Physics, Brighton, 1980), pp. 261.

[8] J. M. Cowley, in Principles of Analytical Electron Microscopy, edited by J. J. Hren, J. I. Goldstein, and D. C. Joy (Plenum, New York, 1986), pp. 343.

[9] S. J. Pennycook, and L. A. Boatner, Nature 336, 565 (1988).

[10] S. J. Pennycook, Ultramic. 30, 58 (1989).

[11] D. H. Shin, E. J. Kirkland, and J. Silcox, Appl. Phys. Lett. 55, 2456 (1989).

[12] S. J. Pennycook, and D. E. Jesson, Phys. Rev. Lett. 64, 938 (1990).

[13] S. J. Pennycook, and D. E. Jesson, Ultramic. 37, 14 (1991).

[14] N. D. Browning, M. F. Chisholm, and S. J. Pennycook, Nature 366, 143 (1993).

[15] P. E. Batson, Nature 366, 727 (1993).

[16] P. E. Batson, N. Dellby, and O. L. Krivanek, Nature 418, 617 (2002).

[17] P. D. Nellist et al., Science 305, 1741 (2004).

[18] M. Varela et al., Phys. Rev. Lett. 92, Art. No. 095502 (2004).

[19] R. Erni et al., Phys. Rev. Lett. 102, Art. No. 096101 (2009).

[20] H. Sawada et al., J. Electron Microsc. 58, 357 (2009).

[21] M. Bosman et al., Phys. Rev. Lett. 99, Art. No. 086102 (2007).

[22] D. A. Muller et al., Science 319, 1073 (2008).

[23] A. J. D'Alfonso et al., Phys Rev B 81 (2010).

[24] M. W. Chu et al., Phys. Rev. Lett. 104 (2010).

[25] O. L. Krivanek et al., Nature 464, 571 (2010).

[26] H. Rose, Optik 39, 416 (1974).

[27] S. D. Findlay et al., Ultramic. 110, 903 (2010).

[28] S. D. Findlay et al., Applied Physics Express 3 (2010).

[29] E. Okunishi et al., Microsc. Microanal. 15, 164 (2009).

[30] R. Ishikawa et al., Nature Mat., 1 (2011).

[31] This work was supported by DOE Office of Science, Office of Basic Energy Sciences, Materials Science and Engineering Division. 